\title{
MODULARIZING THE ELIMINATION OF $r=0$ IN KLEENE ALGEBRA
}

\author{
CHRISTOPHER HARDIN
}

Department of Mathematics, Smith College, Northampton, Massachusetts 01063, USA

e-mail address: chardin@math.smith.edu

\begin{abstract}
Given a universal Horn formula of Kleene algebra with hypotheses of the form $r=0$, it is already known that we can efficiently construct an equation which is valid if and only if the original Horn formula is valid. This is an example of elimination of hypotheses, which is useful because the equational theory of Kleene algebra is decidable while the universal Horn theory is not. We show that hypotheses of the form $r=0$ can still be eliminated in the presence of other hypotheses. This lets us extend any technique for eliminating hypotheses to include hypotheses of the form $r=0$.
\end{abstract}

\section{INTRODUCTION}

Kleene algebra (KA) arises in many areas of computer science, such as automata theory, the design and analysis of algorithms, dynamic logic, and program semantics. Many of these applications are enhanced by using Kleene algebra with tests (KAT), which combines KA with Boolean algebra.

We can use KAT to reason propositionally about programs (see [1, 13, for examples). The equivalence of an optimized and unoptimized program, the equivalence of an annotated and unannotated program, and partial correctness assertions can all be expressed as equations. The equational theory of KAT is well understood and has many useful properties; in particular, it is decidable (in PSPACE) and the theory remains unchanged when we restrict to relational interpretations [4, 14. (Relational interpretations are of the greatest interest because the intended semantics are generally relational.)

However, we frequently wish to reason about programs under certain assumptions about the interaction of atomic programs and tests. For example, if $p$ is the program " $\mathrm{x}:=0$ " and $b$ is the assertion " $\mathrm{x}=0$ ", then we want to be able to make use of the facts $p b=p$ ("after running $p$, test $b$ always succeeds") and $b p=b$ ("after test $b$ succeeds, $p$ is redundant") when reasoning about programs in which $p$ and $b$ appear; for instance, the equation $p^{2}=p$ is not valid in KAT, but the formula $(p b=p \wedge b p=b) \rightarrow p^{2}=p$ is. Thus, the universal Horn theory is of interest. A universal Horn formula is an implication $E \rightarrow s=t$, where $E$ is a finite set of equations. The word "universal" refers to the fact that the atomic symbols of $E, s$, and $t$ are implicitly universally quantified. The universal Horn theory of

2000 ACM Subject Classification: F.3.1.

Key words and phrases: Kleene algebra with tests, program verification, Horn formulas, proof theory. 
a class of structures $\mathrm{C}$, denoted $\mathcal{H C}$, is the set of universal Horn formulas valid under all interpretations over structures in $\mathrm{C}$.

The increased generality of the universal Horn theory is accompanied by greater complexity, and the theory does not remain the same when we restrict to important classes of Kleene algebras such as *-continuous Kleene algebras with tests (KAT*) and relational Kleene algebras with tests (RKAT). HKAT is $\Sigma_{1}^{0}$-complete (undecidable), HKAT* $^{*}$ and $\mathcal{H R K A T}$ are $\Pi_{1}^{1}$-complete (highly undecidable), and we have proper inclusions $\mathcal{H}$ KAT $\subsetneq$ HKAT $^{*} \subsetneq \mathcal{H R K A T}$ [12, 8].

Although these Horn theories are very complex in general, there are fragments of them that are both practical and of lower complexity. Consider the following theorem, fundamentally due to Cohen [2] and extended to the form below by Kozen and Smith [14, 11]. (The statement uses some notions that will not be defined until later, but we only need a vague understanding of it here.)

Theorem 1.1. Let $r, s, t \in \mathrm{RExp}_{\mathrm{P}, \mathrm{B}}$, and let $u \in \mathrm{RExp}_{\mathrm{P}, \mathrm{B}}$ be the universal regular expression. Then the following are equivalent.

$$
\begin{aligned}
\mathrm{KAT} & \models r=0 \rightarrow s=t \\
\mathrm{KAT}^{*} & =r=0 \rightarrow s=t \\
\mathrm{RKAT} & \models r=0 \rightarrow s=t \\
\mathrm{KAT} & =s+u r u=t+u r u
\end{aligned}
$$

The primary consequence of this theorem is that the Horn theory of Kleene algebra, restricted to formulas with hypotheses of the form $r=0$, is decidable, and remains unchanged if we restrict to $*$-continuous or relational algebras: to decide if $r=0 \rightarrow s=t$ is valid, we simply decide if $s+u r u=t+u r u$ is valid. In this way, we say that we have eliminated the hypothesis $r=0$. It is also possible to eliminate other forms of hypotheses 2, 7.

The case $r=0$ has particular significance, because partial correctness assertions can be expressed in KAT with equations of the form $r=0$ (and multiple equations $r_{1}=0 \wedge \cdots \wedge r_{k}=$ 0 can be combined into $r_{1}+\cdots+r_{k}=0$ ). So Theorem 1.1 shows that the Horn theory of KAT, restricted to hypotheses of the form $r=0$, subsumes propositional Hoare logic, is decidable, and is furthermore complete for relational interpretations [11.

Our main result, Theorem [3.2 (p. 9), improves Theorem 1.1] so that $r=0$ can be eliminated in the presence of other hypotheses. This allows any other technique for eliminating hypotheses to be extended to include $r=0$. For example, if we have a technique for eliminating $f=g$ alone, we can eliminate $f=g \wedge r=0$ by first eliminating $r=0$ using Theorem [3.2. leaving hypothesis $f=g$, which can then be eliminated. In this way, Theorem 3.2 is like a module for eliminating $r=0$ that can be added on to any other technique for eliminating hypotheses.

A related result, Corollary 3.10, shows that hypotheses of the form $c p=c$ (where $c$ is Boolean and $p$ is atomic) can be eliminated in the presence of other hypotheses, although the remaining hypotheses are modified. Hypotheses of the form $c p=c$ are useful for eliminating redundant code (consider our example $b p=b$ above; it expresses the fact that $p$ is redundant when $b$ already holds). (The procedure for eliminating $c p=c$ was introduced in [7, where it was shown how to eliminate $c p=c$ and $r=0$ at the same time. Without the benefit of Theorem 3.2 this required a construction that simultaneously dealt with both $c p=c$ and $r=0$. 


\section{Preliminaries}

For a more complete introduction to Kleene algebra and Kleene algebra with tests, see [10].

\subsection{Kleene Algebra.}

Definition 2.1. An idempotent semiring is a structure $(S,+, \cdot, 0,1)$ satisfying

$$
\begin{aligned}
x+x & =x \text { (idempotence) } \\
x+0 & =x \\
x+y & =y+x \\
x+(y+z) & =(x+y)+z \\
0 \cdot x & =x \cdot 0=0 \\
1 \cdot x & =x \cdot 1=x \\
x \cdot(y \cdot z) & =(x \cdot y) \cdot z \\
x \cdot(y+z) & =x \cdot y+x \cdot z \\
(y+z) \cdot x & =y \cdot x+z \cdot x .
\end{aligned}
$$

(In other words, $(S,+, 0)$ is an upper semilattice with bottom element $0,(S, \cdot, 1)$ is a monoid, 0 is an annihilator for $\cdot$, and $\cdot$ distributes over + on the right and left.)

We often write $x y$ for $x \cdot y$. The upper semilattice structure induces a natural partial order on any idempotent semiring: $x \leq y \Leftrightarrow x+y=y$.

Definition 2.2. A Kleene algebra is a structure $\left(K,+, \cdot{ }^{*}, 0,1\right)$ such that $(K,+, \cdot, 0,1)$ forms an idempotent semiring, and which satisfies

$$
\begin{gathered}
1+x x^{*} \leq x^{*} \\
1+x^{*} x \leq x^{*} \\
p+q x \leq x \rightarrow q^{*} p \leq x \\
p+x q \leq x \rightarrow p q^{*} \leq x .
\end{gathered}
$$

(The order of precedence among the operators is ${ }^{*}>\cdot>+$, so that $p+q r^{*}=p+\left(q \cdot\left(r^{*}\right)\right)$.) We let KA denote the category of all Kleene algebras and their homomorphisms. Equations (2.1)-(2.4) are called the Kleene algebra *-axioms.

Given a set $\Sigma$ of constant symbols, let RExp $\operatorname{Re}_{\Sigma}$ be the set of Kleene algebra terms over $\Sigma$. We call the elements of $\operatorname{RExp}_{\Sigma}$ regular expressions, and the elements of $\Sigma$ atomic program symbols. An interpretation is a homomorphism $I: \operatorname{RExp}_{\Sigma} \rightarrow K$, where $K$ is a Kleene algebra. $I$ is determined uniquely by its values on $\Sigma$.

Equations (2.1) and (2.3) say that $q^{*} p$ is the least solution of $p+q x \leq x$, while (2.2) and (2.4) say that $p q^{*}$ is the least solution to $p+x q \leq x$.

A straightforward and vital consequence of the KA axioms ${ }^{1}$ is that the operations + , $\cdot$, and * are monotone: if $x_{0} \leq x_{1}$ and $y_{0} \leq y_{1}$, then $x_{0}+y_{0} \leq x_{1}+y_{1}, x_{0} y_{0} \leq x_{1} y_{1}$, and $x_{0}^{*} \leq x_{1}^{*}$.

\footnotetext{
${ }^{1}$ The names of the categories we consider serve as convenient abbreviations for the type of algebra they contain. So, for example, "the KA axioms" means "the axioms of Kleene algebra".
} 
We use $\models$ to denote ordinary Tarskian satisfaction. However, since we have constant symbols from $\Sigma$ not in the signatures of the underlying algebras, we will pair each algebra with an interpretation when speaking about satisfaction. For example, given a Kleene algebra $K$, interpretation $I: \operatorname{RExp}_{\Sigma} \rightarrow K$, and formula $\varphi$ whose atomic program symbols are among $\Sigma$, we will write $K, I \models \varphi$ to indicate that $K$ satisfies $\varphi$ when the symbols in $\Sigma$ are evaluated according to $I . K \models \varphi$ means that $K, I \models \varphi$ for every interpretation $I: \operatorname{RExp}_{\Sigma} \rightarrow K$. We also use $\models$ in two other standard ways: for a class $C$ of algebras, $\mathrm{C} \models \varphi$ means that $K \models \varphi$ for each $K \in \mathrm{C}$; for a set $\Phi$ of formulas, $\Phi \models \varphi$ means that $K \models \varphi$ for each algebra $K$ satisfying every formula in $\Phi$.

We now introduce two particularly important types of Kleene algebras: language algebras and relational algebras.

Definition 2.3. For an arbitrary monoid $M$, its powerset $2^{M}$ forms a Kleene algebra as follows.

$$
\begin{aligned}
0 & =\varnothing \\
1 & =\left\{1^{M}\right\}\left(\text { where } 1^{M} \text { is the identity of } M\right) \\
A+B & =A \cup B \\
A \cdot B & =\{x y \mid x \in A, y \in B\} \\
A^{*} & =\bigcup_{k \in \mathbb{N}} A^{k}
\end{aligned}
$$

We let REG $M$ denote the smallest subalgebra of $2^{M}$ containing the singletons $\{x\}, x \in M$. (The elements of REG $M$ are the regular subsets of $M$.) $2^{M}$ and its subalgebras are known as language algebras.

Of particular interest is the case $M=\Sigma^{*}$, the monoid of all strings over alphabet $\Sigma$ under concatenation. The empty string $\varepsilon$ is the identity of this monoid. We define the canonical interpretation $R: \operatorname{RExp}_{\Sigma} \rightarrow \operatorname{REG} \Sigma^{*}$ by letting $R(p)=\{p\}$ (and extending $R$ homomorphically to the rest of $\operatorname{RExp}{ }_{\Sigma}$ ). Note that we can interpret elements of $\Sigma^{*}$ as elements of $\operatorname{RExp}_{\Sigma}$ in the obvious fashion.

Definition 2.4. For an arbitrary set $X$, the set $2^{X \times X}$ of all binary relations on $X$ forms a Kleene algebra as follows.

$$
\begin{aligned}
0 & =\varnothing \\
1 & =\iota_{X}=\{(x, x) \mid x \in X\} \\
S+T & =S \cup T \\
S \cdot T & =S \circ T(\text { the composition of } S \text { with } T) \\
S^{*} & =\bigcup_{k \in \mathbb{N}} S^{k}(\text { the reflexive transitive closure of } S)
\end{aligned}
$$

A Kleene algebra $K$ is relational if it is a subalgebra of $2^{X \times X}$ for some $X ; X$ is called the base of $K$. We let RKA denote the category of all relational Kleene algebras and their homomorphisms.

The definitions of * in $2^{M}$ and $2^{X \times X}$ exemplify the most common intuition about the meaning of ${ }^{*}$, which is that $y^{*}=\sup _{n \in \mathbb{N}} y^{n}$, or informally, $y^{*}=1+y+y^{2}+\cdots$. (More generally, if we require that multiplication distributes over this supremum, we have 
$x y^{*} z=x 1 z+x y z+x y^{2} z+\cdots=\sup _{n \in \mathbb{N}} x y^{n} z$.) However, this property of * does not follow from the KA $*$-axioms, and must be postulated separately.

Definition 2.5. A Kleene algebra $K$ is $*$-continuous if it satisfies

$$
x y^{*} z=\sup _{k \in \mathbb{N}} x y^{k} z
$$

for all $x, y, z \in K$. We let $\mathrm{KA}^{*}$ denote the category of all $*$-continuous Kleene algebras and their homomorphisms.

Since relational composition distributes over arbitrary union, it is immediate from the definition of * in $2^{X \times X}$ that relational Kleene algebras are $*$-continuous, so RKA $\subseteq \mathrm{KA}^{*}$.

The following ubiquitous lemma is a useful generalization of $*$-continuity.

Lemma 2.6. Suppose $K \in \mathrm{KA}^{*}, I: \mathrm{RExp}_{\Sigma} \rightarrow K$ is an interpretation, and $t \in \mathrm{RExp}_{\Sigma}$. Then

$$
I(t)=\sup _{\sigma \in R(t)} I(\sigma)
$$

Proof. By induction on structure of $t$. For details, see [9, Lemma 7.1, pp. 246-248].

2.2. Kleene Algebra with Tests. We can combine Kleene algebra with Boolean algebra to get Kleene algebra with tests. The Boolean aspect is useful for capturing Boolean aspects of programming semantics, particularly control flow and assertions.

Definition 2.7. A Kleene algebra with tests is a two-sorted structure $\left(K, B,+, \cdot{ }^{*},{ }^{-}, 0,1\right)$, where $\left(K,+, \cdot{ }^{*}, 0,1\right)$ is a Kleene algebra, and $\left(B,+, \cdot{ }^{-}, 0,1\right)$ is a Boolean subalgebra. The elements of $B$ are called tests. We let KAT denote the category of all Kleene algebras with tests and their homomorphisms; we let $\mathrm{KAT}^{*}$ denote the subcategory of all $*$-continuous Kleene algebras with tests.

We now have two types of atomic symbols: programs and tests. For a finite set $\mathrm{P}$ of atomic program symbols and a finite set $B$ of atomic test symbols, RExp $p_{\mathrm{P}, \mathrm{B}}$ is the set of KAT terms over $\mathrm{P}$ and $\mathrm{B}$; negation can only be applied to Boolean terms, which are terms built from $0,1,+, \cdot$, , and atomic test symbols. An interpretation $I: \operatorname{RExp}_{\mathrm{P}, \mathrm{B}} \rightarrow K$ must map each atomic test to a test in $K$ (and it follows by induction that it will map all Boolean terms to tests).

$2^{X \times X}$ forms a Kleene algebra with tests by keeping the previously defined Kleene algebra structure, and letting $B=\left\{r \in 2^{X \times X} \mid r \leq 1\right\}, \bar{b}=\iota_{X}-b$. A Kleene algebra with tests $K$ is relational if it is a subalgebra of $2^{X \times X}$ for some $X$. We let RKAT denote the category of all relational Kleene algebras with tests and their homomorphisms.

Every Kleene algebra induces a Kleene algebra with tests by letting $B=\{0,1\}$, the two-element Boolean algebra; conversely, every Kleene algebra with tests induces a Kleene algebra by taking its reduct to the signature of Kleene algebra (i.e., taking its image under the map $\left.\left(K, B,+, \cdot,^{*},{ }^{-}, 0,1\right) \mapsto\left(K,+, \cdot{ }^{*}, 0,1\right)\right)$. With this in mind, it is easy to see that for any formula $\varphi$ in the language of Kleene algebra, $\mathrm{KAT} \models \varphi \Leftrightarrow \mathrm{KA} \models \varphi, \mathrm{KAT}^{*} \models \varphi \Leftrightarrow$ $\mathrm{KA}^{*} \models \varphi$, and $\mathrm{RKAT} \models \varphi \Leftrightarrow \mathrm{RKA} \models \varphi$.

There is an analog of REG $\Sigma^{*}$ for KAT called the guarded-string model, with its own analog of the canonical interpretation $R$. Though the guarded-string model is in general very important for studying KAT, we will not need it for our results here, and refer the reader to 14] for further information on guarded strings.

The following elementary lemma about subalgebras will be needed in Lemma 3.3 . 
Lemma 2.8. Let $K \in \mathrm{KA}$ and let $x \in K$. Then $\{y \in K \mid y \leq x\}$ is a subalgebra of $K$ iff $x=y^{*}$ for some $y \in K$ (or equivalently, $x=x^{*}$ ). The same also holds for KATs. (Note that this is not claiming that all subalgebras of $K$ have this form.)

The proof is straightforward and may safely be skipped.

Proof. Let $K^{\prime}=\{y \in K \mid y \leq x\}$.

Suppose $K^{\prime}$ is a subalgebra of $K$. Then $x^{*} \in K^{\prime}$, so $x^{*} \leq x$, so $x=x^{*}$.

Suppose $x=y^{*}$ for some $y \in K$. Then $x^{*}=y^{* *}=y^{*}=x$. The necessary closure conditions follow from monotonicity and the fact that $0+1+x x+(x+x)+x^{*} \leq x^{*}$. (For example, for any $y_{1}, y_{2} \in K^{\prime}$, we have $y_{1} y_{2} \leq x x \leq x^{*}$.)

\subsection{Universal Horn Formulas.}

Definition 2.9. A universal Horn formula is a formula of the form

$$
s_{1}=t_{1} \wedge \cdots \wedge s_{t}=t_{k} \rightarrow s=t,
$$

where $s_{i}, t_{i}, s, t$ are terms. The set of universal Horn formulas valid over a class $\mathrm{C}$ of algebras is the universal Horn theory of $\mathrm{C}$, which we denote by $\mathcal{H C}$.

We will often drop the word "universal". Note that in KA and KAT, because any inequality $x \leq y$ is actually an equation $x+y=y$, inequalities are allowed in Horn formulas. We will allow finite sets of equations to appear in the hypotheses of a Horn formula, by taking their conjunction; e.g., if $E=\{p q=q p, p \leq 1\}$, then $E \rightarrow s=t$ means $(p q=$ $q p \wedge p \leq 1) \rightarrow s=t$.

Lemma 2.10. Let $\Gamma$ be any class of $*$-continuous Kleene algebras with interpretations. (That is, $\Gamma$ consists of pairs $(K, I)$ where $K \in \mathrm{KA}^{*}$ and $I: \operatorname{RExp}_{\Sigma} \rightarrow K$ is an interpretation.) Then for any Horn formula of the form $E \rightarrow s \leq t$,

$$
\Gamma \models E \rightarrow s \leq t \Longleftrightarrow(\forall \sigma \in R(s)) \Gamma \models E \rightarrow \sigma \leq t .
$$

Proof. For any $K \in \mathrm{KA}^{*}$ with interpretation $I: \operatorname{RExp}_{\Sigma} \rightarrow K$, the equivalence

$$
K, I \models E \rightarrow s \leq t \Longleftrightarrow(\forall \sigma \in R(s)) K, I \models E \rightarrow \sigma \leq t
$$

is a straightforward consequence of Lemma 2.6. The lemma then follows by exchanging the universal quantifiers $(\forall \sigma \in R(s))$ and $(\forall(K, I) \in \Gamma)$. (This latter quantifier comes from $\Gamma \models E \rightarrow s \leq t \Leftrightarrow(\forall(K, I) \in \Gamma) K, I \models E \rightarrow s \leq t$. $)$

2.4. A Proof System for $\mathcal{H}$ RKA. Later, in the proof of Lemma 3.5, we will use a prooftheoretic argument based on the infinitary proof system for $\mathcal{H}$ RKA introduced in [6]. We will only present the material that we will need in Section 3.1 for the proof of Lemma 3.5. for a more thorough treatment, please see [6].

2.4.1. Finite Automata and Trees. Our proof system for $\mathcal{H R K A}$ is based on trees of finite automata, and we must define a number of notions related to trees and automata before continuing.

Assume we have a fixed finite alphabet $\Sigma$. We let NFA denote the set of all nondeterministic finite automata over $\Sigma$, allowing $\varepsilon$-moves (also called $\varepsilon$-edges).

We will also use NFA as shorthand for nondeterministic finite automaton. For any NFA $A, L(A)$ denotes the language of $A$, and $|A|$ denotes the states of $A$. For states $v, w \in|A|$, let $A^{v, w}$ denote the NFA which is identical to $A$ except that it has $v$ and $w$ as its unique 
start and accept states, respectively. We fix distinct states $a$ and $b$, and let $\mathrm{NFA}^{a, b}$ be the set of all $A \in$ NFA which have unique start state $a$ and unique accept state $b$.

We define $F_{0} \in \mathrm{NFA}^{a, b}$ to have states $\{a, b\}$ and no edges.

Given an NFA $A$ and states $v, w \in|A|$, we will sometimes want to "insert" a string $\tau \in \Sigma^{*}$ into $L\left(A^{v, w}\right)$. For this purpose, we define $A^{\prime}=\operatorname{insert}_{2}(A, v, w, \tau)$ as follows.

(1) If $\tau=p_{1} \cdots p_{k}$, with $p_{i} \in \Sigma$ and $k>0$, we obtain $A^{\prime}$ from $A$ by adding $k-1$ new states $x_{1}, \ldots, x_{k-1}$ and adding edges

$$
v \stackrel{p_{1}}{\rightarrow} x_{1} \stackrel{p_{2}}{\rightarrow} \cdots \stackrel{p_{k-1}}{\rightarrow} x_{k-1} \stackrel{p_{k}}{\rightarrow} w
$$

(2) If $\tau=\varepsilon$, then we add an $\varepsilon$-edge from $v$ to $w$ and also from $w$ to $v$. (Where it is used, insert ${ }_{2}(A, v, w, \varepsilon)$ corresponds to identifying $v$ and $w$ with each other. The edge from $w$ to $v$, called a reverse $\varepsilon$-edge, is needed to capture the symmetry of the identity relation.)

We now move on to trees. $\mathbb{N}^{*}$ is the set of all finite strings of naturals (including the empty string). A set $T \subseteq \mathbb{N}^{*}$ is a tree if it is closed under taking initial segments. A function $f: \mathbb{N} \rightarrow \mathbb{N}$ can be treated as an infinite sequence of naturals, and for $n \in \mathbb{N}$, we let $f \uparrow n$ denote the initial segment of $f$ of length $n$. Such an $f$ is a path through a tree $T$ if $(f\lceil n) \in T$ for all $n \in \mathbb{N}$. (We find this a concise framework for countably-branching trees, but it is not strictly necessary to define trees in this manner.)

2.4.2. Relational Proofs. The following definition of relational proof captures, with trees of finite automata, the combinatorics of attempting to construct a relational counterexample to a Horn formula. A path through such a tree yields a relational model in which the formula fails, while well-foundedness establishes the impossibility of a counterexample (i.e., the relational validity of the formula).

Definition 2.11. Let $E \rightarrow \sigma \leq t$ be a Horn formula in the language of KA with $\sigma \in \Sigma^{*}$ and $t \in \operatorname{RExp}_{\Sigma}$. We assume that all hypotheses in $E$ are inequalities $x \leq y$, by breaking any equations $x=y$ into $x \leq y \wedge y \leq x$ as necessary. We fix distinct states $a$ and $b$ as above. We fix a special symbol CON, which will signify contradiction.

A relational tree for $E \rightarrow \sigma \leq t$ is a pair $(T, A)$ where $T \subseteq \mathbb{N}^{*}$ is a tree and $A: T \rightarrow$ $\mathrm{NFA}^{a, b} \cup\{\mathrm{CON}\}$ such that the following conditions hold. ( $A_{f}$ will denote $A(f)$.)

(1) At the root, we have $A_{\langle\rangle}=\operatorname{insert}_{2}\left(F_{0}, a, b, \sigma\right)$.

(2) $f \in T$ is a leaf node if and only if $A_{f}=\mathrm{CON}$ or $R(t) \cap L\left(A_{f}\right) \neq \varnothing$.

(3) If $f$ is not a leaf node, then there exist $v, w \in\left|A_{f}\right|$ (possibly equal), an inequality $r \leq r^{\prime}$ in $E$, and $\rho \in L\left(A_{f}^{v, w}\right) \cap R(r)$ such that

(a) if $R\left(r^{\prime}\right)=\varnothing$ (typically because $r^{\prime}=0$ ), then $f$ has one child $g$, with $A_{g}=\mathrm{CON}$;

(b) if $R\left(r^{\prime}\right) \neq \varnothing$, then $f$ has one child $g_{\tau}$ for each $\tau \in R\left(r^{\prime}\right)$, with $A_{g_{\tau}}=$ insert $_{2}\left(A_{f}, v, w, \tau\right)$.

(We say that the hypothesis $r \leq r^{\prime}$ is applied at $f$.)

A relational proof of $E \rightarrow \sigma \leq t$ is a well-founded relational tree for $E \rightarrow \sigma \leq t$. We say $E \rightarrow \sigma \leq t$ is relationally provable if such a proof exists.

Lemma 2.12. For any Horn formula of the form $E \rightarrow \sigma \leq t$, the following are equivalent.

(i) $\mathrm{RKA} \models E \rightarrow \sigma \leq t$

(ii) $E \rightarrow \sigma \leq t$ is relationally provable.

Proof. See [5] or [6]. 
The notion of relational provability can be extended to arbitrary Horn formulas, but we will not need it for the proof of Lemma 3.5.

2.5. The Relationship Between HRKA and HRKAT. The system presented in Section 2.4 is a tool for studying $\mathcal{H}$ RKA, while in Lemma 3.5 we will wish to use it to draw conclusions about $\mathcal{H}$ RKAT. This must be rectified, and there are multiple ways to proceed. One would be to modify the notion of relational proof so that it applies to HRKAT; this would present no particular difficulty, but would require a closer look at relational proofs than we would like to get into here. Instead, we will show how to reduce questions about $\mathcal{H}$ RKAT to $\mathcal{H}$ RKA, in a way that will allow us to use the existing definition of relational proof when proving Lemma 3.5

Lemma 2.13. For any Horn formula $\varphi$ of KAT, there is a Horn formula $\operatorname{Tr}(\varphi)$ of KA such that $\mathrm{RKAT} \models \varphi$ iff $\mathrm{RKA} \models \operatorname{Tr}(\varphi)$.

The lemma is uninteresting without putting restrictions on the translation Tr. However, instead of trying to capture the desired properties of $\operatorname{Tr}$ for inclusion in the lemma, we just give the proof, and observe later that the translation works for a particular purpose when the need arises.

Proof. (Outline: we first assume that negation is only applied to atomic tests, then replace the negations of atomic tests with fresh program symbols, and finally add new hypotheses to ensure that the new program symbols behave like the negated tests they replace.)

Fix a set $\mathrm{P}$ of atomic program symbols, and a set $\mathrm{B}$ of atomic tests. Given any $s \in$ $\operatorname{RExp}_{\mathrm{P}, \mathrm{B}}$, we can assume without loss of generality that negation is only applied to atomic tests, in light of DeMorgan's Laws.

For each $b \in \mathrm{B}$, we introduce two new atomic program symbols $\tilde{b}$ and $\tilde{\bar{b}}$, and we let $\Sigma=\mathrm{P} \bigcup\{\tilde{b}, \tilde{\bar{b}} \mid b \in \mathrm{B}\}$. For any $t \in \operatorname{RExp}_{\mathrm{P}, \mathrm{B}}$, we let $\tilde{t}$ be the result of taking $t$, and replacing all occurrences of $\bar{b}$ with $\tilde{\bar{b}}$, and all positive occurrences of $b$ with $\tilde{b}$ (for each $b \in \mathrm{B}$ ). Note that $\tilde{t} \in \operatorname{RExp}_{\Sigma}$. For any formula $\varphi$, we let $\tilde{\varphi}$ be the result of replacing each term $t$ in $\varphi$ with $\tilde{t}$.

Now take any Horn formula $\varphi$ of the form $\theta \rightarrow \psi$ (with all terms in $\operatorname{RExp} \operatorname{Pe}_{\mathrm{P}, \mathrm{B}}$ ). Let $\operatorname{Tr}(\varphi)$ be the formula

$$
\left(\tilde{\theta} \wedge \bigwedge_{b \in \mathrm{B}}(\tilde{b}+\tilde{\bar{b}}=1 \wedge \tilde{b} \cdot \tilde{\bar{b}}=0)\right) \rightarrow \tilde{\psi}
$$

(The extra hypotheses make $\tilde{b}$ and $\tilde{\bar{b}}$ behave like Boolean complements of each other.)

We now show RKAT $\models \varphi$ iff $\operatorname{RKA} \models \operatorname{Tr}(\varphi)$.

For the right-to-left implication, suppose RKAT $\forall \varphi$. Let $K \in$ RKAT with interpretation $I: \operatorname{RExp}_{\mathrm{P}, \mathrm{B}} \rightarrow K$ such that $K, I \forall \models \varphi$. Then $K, I \models \theta \wedge \neg \psi$. Define the interpretation $\tilde{I}: \operatorname{RExp}_{\Sigma} \rightarrow K$ by

$$
\tilde{I}(p)= \begin{cases}I(p), & \text { if } p \in \mathbf{P} \\ I(b), & \text { if } p=\tilde{b} \\ I(\bar{b}), & \text { if } p=\tilde{\bar{b}}\end{cases}
$$


A simple induction shows that for any $t \in \operatorname{RExp}_{\mathrm{P}, \mathrm{B}}, \tilde{I}(\tilde{t})=I(t)$. It follows that $K, \tilde{I} \models \tilde{\theta} \wedge \neg \tilde{\psi}$, since $K, I \models \theta \wedge \neg \psi$. Also,

$$
K, \tilde{I} \models \bigwedge_{b \in \mathrm{B}}(\tilde{b}+\tilde{\bar{b}}=1 \wedge \tilde{b} \cdot \tilde{\bar{b}}=0) .
$$

Thus $K, \tilde{I} \not \models \operatorname{Tr}(\varphi)$, so RKA $\not \models \operatorname{Tr}(\varphi)$ (recall that we can treat $K$ as a member of RKA by passing it through the forgetful functor which drops negation). Therefore, RKA $\models$ $\operatorname{Tr}(\varphi) \rightarrow \operatorname{RKAT} \models \varphi$.

For the left-to-right implication, suppose that RKA $\forall \neq \operatorname{Tr}(\varphi)$. Let $K \in \operatorname{RKA}$ with interpretation $I: \operatorname{RExp}_{\Sigma} \rightarrow K$ such that $K, I \not \forall \operatorname{Tr}(\varphi)$. Let $X$ be the base of $K$. Then $K \subseteq 2^{X \times X}$, so $2^{X \times X}, I \not \models \operatorname{Tr}(\varphi)$; that is,

$$
2^{X \times X}, I \models \tilde{\theta} \wedge \bigwedge_{b \in \mathrm{B}}(\tilde{b}+\tilde{\bar{b}}=1 \wedge \tilde{b} \cdot \tilde{\bar{b}}=0) \wedge \neg \tilde{\psi} .
$$

In particular, for any $b \in \mathrm{B}, I(\tilde{b}) \cup I(\tilde{\bar{b}})=I(1)$, and $I(\tilde{b}) \circ I(\tilde{\bar{b}})=\varnothing$; it follows that $I(\tilde{b}) \bigcap I(\tilde{\bar{b}})=\varnothing($ since $R \bigcap S=R \circ S$ whenever $R, S \subseteq I(1))$, so $I(\overline{\bar{b}})=I(1)-I(\tilde{b})$.

Define the interpretation $I^{\prime}: \operatorname{RExp}_{\mathrm{P}, \mathrm{B}} \rightarrow 2^{X \times X}$ by

$$
\begin{aligned}
I^{\prime}(p) & =I(p), \\
I^{\prime}(b) & =I(\tilde{b}) .
\end{aligned}
$$

We have

$$
\begin{aligned}
I^{\prime}(\bar{b}) & =I^{\prime}(1)-I^{\prime}(b) \\
& =I(1)-I(\tilde{b}) \\
& =I(\tilde{\bar{b}}) .
\end{aligned}
$$

It follows that, for any $t \in \operatorname{RExp}_{\mathrm{P}, \mathrm{B}}, I^{\prime}(t)=I(\tilde{t})$. So, $2^{X \times X}, I^{\prime} \models \theta \wedge \neg \psi$, since $2^{X \times X}, I \models$ $\tilde{\theta} \wedge \neg \tilde{\psi}$, giving us RKAT $\forall \varphi$. Therefore, RKAT $\models \varphi \rightarrow \operatorname{RKA} \models \operatorname{Tr}(\varphi)$, completing the proof.

\section{Main Results}

\subsection{Eliminating $r=0$.}

Definition 3.1. For a fixed set $\mathrm{P}=\left\{p_{1}, \ldots, p_{n}\right\}$ of atomic program symbols, the universal regular expression $u$ is defined by

$$
u=\left(p_{1}+\cdots+p_{n}\right)^{*} .
$$

We trivially have $\mathrm{KAT} \models u=u u=u^{*}$, and a straightforward induction shows that, for any $s \in \mathrm{RExp}_{\mathrm{P}, \mathrm{B}}$, KAT $\models s \leq u$.

Our goal is the following theorem.

Theorem 3.2. Let $u$ be the universal regular expression, let $E$ be any finite set of hypotheses, and let $r, s, t \in \mathrm{RExp}_{\mathrm{P}, \mathrm{B}}$. Then the following equivalences hold.

$$
\begin{aligned}
& \mathrm{KAT} \models E \wedge r=0 \rightarrow s=t \Longleftrightarrow \mathrm{KAT} \models E \rightarrow s+u r u=t+u r u \\
& \mathrm{KAT}^{*} \models E \wedge r=0 \rightarrow s=t \Longleftrightarrow \mathrm{KAT}^{*} \models E \rightarrow s+u r u=t+u r u \\
& \mathrm{RKAT} \models E \wedge r=0 \rightarrow s=t \Longleftrightarrow \mathrm{RKAT} \models E \rightarrow s+u r u=t+u r u
\end{aligned}
$$


Note that the special case $E=\varnothing$ is essentially Theorem 1.1 (when $E=\varnothing$, the right hand sides of (3.1)-(3.3) are equivalent, since the equational theories of KAT, KAT*, and RKAT coincide; when $E \neq \varnothing$, the right hand sides of (3.1)-(3.3) are no longer necessarily equivalent, which prevents Theorem 3.2 from having the same form as Theorem 1.1. Note also that for any formula $\varphi$ in the language of $\mathrm{KA}$, we have $\mathrm{KA} \models \varphi$ iff $\mathrm{KAT} \models \varphi, \mathrm{KA}^{*} \models \varphi$ iff $\mathrm{KAT}^{*} \models \varphi$, etc., so Theorem 3.2 also applies to KA, KA*, and RKA. (Alternatively, omitting the Boolean aspects of the proof that follows would yield a proof of the analogous theorem for $\mathrm{KA}, \mathrm{KA}^{*}$, and RKA.)

We prove each equivalence as a separate lemma. Fix $u, E, r, s, t$, as above.

Lemma 3.3.

$$
\mathrm{KAT} \models E \wedge r=0 \rightarrow s=t \Longleftrightarrow \mathrm{KAT} \models E \rightarrow s+u r u=t+u r u
$$

Proof. The right-to-left implication is trivial: reasoning under $E \wedge r=0$, we have $s=$ $s+0=s+u r u=t+u r u=t+0=t$. (Note that this argument also applies to KAT $^{*}$ and RKAT.)

For the left-to-right implication, suppose $\mathrm{KAT} \models E \wedge r=0 \rightarrow s=t$. Take any $K \in \mathrm{KAT}$ with interpretation $I$ such that $K, I \models E$. Let $\perp=I(u r u), \top=I(u)$, noting that $\top^{*}=\top$, $\perp=\top \perp=\perp \top$, and $\perp \perp \leq \perp$. Let $K^{\prime}=\{x \in K \mid x \leq \top\}$. This is a subalgebra of $K$ by Lemma 2.8 since $\top=\top^{*} . I$ is an interpretation into $K^{\prime}$.

Define the map $f: K^{\prime} \rightarrow K^{\prime}$ by $f(x)=x+\perp$. Let $L=f\left[K^{\prime}\right]$, the image of $K^{\prime}$ under $f$. $\top$ and $\perp$ are respectively the greatest and least elements of $L$. Note that for any $x \in K^{\prime}$, $x \top \leq \top$, so $x \perp=x \top \perp \leq \top \perp=\perp$. We similarly have $\perp x \leq \perp$.

Define

$$
\begin{aligned}
0^{L} & =\perp=f(0) \\
1^{L} & =1+\perp=f(1) \\
v \cdot{ }^{L} w & =v \cdot w+\perp=f(v w) .
\end{aligned}
$$

Let $L$ be the structure $\left(L, f[B],+,{ }^{L},{ }^{*}, \sim, 0^{L}, 1^{L}\right)$, in the signature of KAT, where $B$ is the set of tests of $K^{\prime}$, and the Boolean complement $\sim$ is defined by $\widetilde{f(c)}=f(\bar{c})$. We must show that ${ }^{\sim}$ is well-defined. Suppose $f(c)=f(d)$. Then

$$
\begin{aligned}
f(\bar{c}) & =\bar{c}+\perp \\
& \leq(\bar{c}+\perp)(1+\perp) \\
& =(\bar{c}+\perp)(d+\bar{d}+\perp) \\
& =(\bar{c}+\perp)(c+\bar{d}+\perp) \quad(\text { since } c+\perp=f(c)=f(d)=d+\perp) \\
& =\bar{c} c+\bar{c} \bar{d}+\bar{c} \perp+\perp c+\perp \bar{d}+\perp \perp \\
& \leq 0+\bar{d}+\perp \\
& =f(\bar{d}) .
\end{aligned}
$$

Similarly, $f(\bar{d}) \leq f(\bar{c})$, so $f(\bar{c})=f(\bar{d})$. Therefore, $\sim$ is well-defined.

We claim that $f: K^{\prime} \rightarrow L$ is a homomorpishm. (Note that this is different from claiming that $f: K^{\prime} \rightarrow K^{\prime}$ is a homomorphism, which is not true unless $\perp=0$.) For any 
$x, y \in K$, and $c$ a test in $K$,

$$
\begin{aligned}
f(0) & =0^{L} \\
f(1) & =1^{L} \\
f(x+y) & =x+y+\perp=x+\perp+y+\perp=f(x)+f(y) \\
f(x y) & =x y+\perp \\
& =x y+\perp y+x \perp+\perp \perp+\quad(\text { since } \perp y+x \perp+\perp \perp \leq \perp) \\
& =(x+\perp)(y+\perp)+\perp \\
& =f(x) \cdot{ }^{L} f(y) \\
f(\bar{c}) & =\widetilde{f(c)} .
\end{aligned}
$$

It remains to verify $f\left(x^{*}\right)=(f(x))^{*}$. We have

$$
1+(x+\perp)\left(x^{*}+\perp\right)=1+x x^{*}+x \perp+\perp x^{*}+\perp \perp \leq x^{*}+\perp,
$$

so the $*$-axioms give us $(x+\perp)^{*} \leq x^{*}+\perp$. We have $x^{*} \leq(x+\perp)^{*}$ and $\perp \leq(x+\perp)^{*}$ trivially, so $x^{*}+\perp \leq(x+\perp)^{*}$. Therefore, $f\left(x^{*}\right)=x^{*}+\perp=(x+\perp)^{*}=(f(x))^{*}$. So $f: K^{\prime} \rightarrow L$ is a homomorphism.

We now claim that $L \in$ KAT. Since $f: K^{\prime} \rightarrow L$ is a homomorphism and $K^{\prime} \in \mathrm{KAT}, L$ automatically satisfies the equational KAT axioms. We must now verify that $L$ satisfies the two remaining axioms, $p+q \cdot{ }^{L} x \leq x \rightarrow q^{*} \cdot{ }^{L} p \leq x$ and $p+x \cdot{ }^{L} q \leq x \rightarrow p \cdot{ }^{L} q^{*} \leq x$.

Suppose that $p+q \cdot{ }^{L} x \leq x$. We must show $q^{*} \cdot{ }^{L} p \leq x$. We have $p+q x+\perp=$ $p+q \cdot{ }^{L} x \leq x$. From $p+q x \leq x$ we conclude $q^{*} p \leq x$; combining this with $\perp \leq x$, we have $q^{*} \cdot{ }^{L} p=q^{*} p+\perp \leq x$, as desired. Similarly, $p+x \cdot{ }^{L} q \leq x \rightarrow p \cdot{ }^{L} q^{*} \leq x$. So $L \in \mathrm{KAT}$.

Define the interpretation $J: \operatorname{RExp}_{\mathrm{P}, \mathrm{B}} \rightarrow L$ by $J(q)=f(I(q))$. Since $K^{\prime}, I \models E$, it immediately follows that $L, J \models E$. Also, $J(r) \leq J($ uru $)=f(I($ uru $))=f(\perp)=\perp+\perp=$ $0^{L}$, so $L, J \models r=0$. Therefore, the assumption KAT $\models E \wedge r=0 \rightarrow s=t$ gives us $L, J \models s=t$. Therefore,

$$
I(s+u r u)=I(s)+I(u r u)=I(s)+\perp=f(I(s))=J(s)=J(t)=I(t+u r u) .
$$

Thus, $K, I \models s+u r u=t+u r u$.

\section{Lemma 3.4.}

$$
\mathrm{KAT}^{*} \models E \wedge r=0 \rightarrow s=t \Longleftrightarrow \mathrm{KAT}^{*} \models E \rightarrow s+u r u=t+u r u
$$

Proof. The right-to-left implication is as in Lemma 3.3

For the left-to-right implication, it suffices to verify that the construction in the proof of Lemma 3.3 preserves $*$-continuity. Letting $q^{(n)}$ denote the $n^{\text {th }}$ power of $q$ under ${ }^{L}$ (with $\left.q^{(0)}=1^{L}\right)$, we have

$$
\begin{aligned}
\sup _{n} p \cdot{ }^{L} q^{(n)} \cdot{ }^{L} r & =\sup _{n}\left(p q^{n} r+\perp\right) \\
& =p q^{*} r+\perp \\
& =p \cdot{ }^{L} q^{*} \cdot{ }^{L} r .
\end{aligned}
$$

(For the second equality above, one can observe that $p q^{n} r+\perp \leq p q^{*} r+\perp$ for all $n$, and that if $x$ is any upper bound for $p q^{n} r+\perp$, then $p q^{*} r=\sup _{n} p q^{n} r \leq x$ and $\perp \leq x$, so $p q^{*} r+\perp \leq x$. So $\sup _{n}\left(p q^{n} r+\perp\right)=p q^{*} r+\perp$.) 


\section{Lemma 3.5.}

$$
\mathrm{RKAT} \models E \wedge r=0 \rightarrow s=t \Longleftrightarrow \mathrm{RKAT} \models E \rightarrow s+u r u=t+u r u
$$

Proof. The right-to-left implication is as in Lemma 3.3

For the left-to-right implication, using the above construction would require verifying that $L$ has a relational representation, which is not clear. Instead, we use a proof-theoretic argument. Suppose RKAT $\models E \wedge r=0 \rightarrow \sigma \leq t$, where $\sigma \in R(s) . r=0$ is equivalent to $r \leq 0$, and $\mathrm{KAT} \models t \leq t+u r u$, so RKAT $\models E \wedge r \leq 0 \rightarrow \sigma \leq t+u r u$.

For the moment, suppose that the formulas are in the language of $\mathrm{KA}$, so that we can speak about relational proofs without worrying about tests. Let $(T, A)$ be a relational proof of $E \wedge r \leq 0 \rightarrow \sigma \leq t+u r u$.

We claim that the hypothesis $r \leq 0$ is never even applied in the proof! Suppose $r \leq 0$ is applied at node $f \in T$ (so $f$ has one child $g$ with $A_{g}=\mathrm{CON}$ ). For $r \leq 0$ to be applied at $f$, there must be states $v, w \in\left|A_{f}\right|$ and $\rho \in R(r)$ with $\rho \in L\left(A_{f}^{v, w}\right)$. A property that is preserved in the automata of relational trees is that every state is accessible from the start state $a$, and the accept state $b$ is accessible from every state. So there exist $\pi \in L\left(A_{f}^{a, v}\right)$ and $\pi^{\prime} \in L\left(A_{f}^{w, b}\right)$. Thus, we have $\pi \rho \pi^{\prime} \in L\left(A_{f}\right)$; we also have $\pi \rho \pi^{\prime} \in R(u r u) \subseteq R(t+u r u)$. Therefore, $R(t+u r u) \cap L\left(A_{f}\right) \neq \varnothing$, so $f$ is in fact a leaf node, contradicting the assumption that we are applying $r \leq 0$ at $f$. (In other words, at any point in a relational tree for $E \wedge r \leq 0 \rightarrow \sigma \leq t+u r u$ where we could apply $r \leq 0$, we would already have to be at a leaf.)

So, because $r \leq 0$ is never applied, $(T, A)$ is also a relational proof of $E \rightarrow \sigma \leq t+u r u$. Therefore, RKA $=E \rightarrow \sigma \leq t+u r u$ for all $\sigma \in R(s)$. By Lemma 2.10. RKA $\models E \rightarrow s \leq t+u r u$, so $\mathrm{RKA} \models E \rightarrow s+u r u \leq t+u r u$. RKA $\models E \rightarrow t+u r u \leq s+u r u$ is similar, and we now have $\mathrm{RKA}=E \rightarrow s+u r u=t+u r u$.

In case the formulas are not in the language of $K A$, we can use the translation from Section 2.5 as follows. We use the above argument to get

$$
\mathrm{RKA} \models \operatorname{Tr}(E \wedge r=0 \rightarrow s=t) \Rightarrow \mathrm{RKA} \models \operatorname{Tr}(E \rightarrow s+u r u=t+u r u) .
$$

(The extra hypotheses introduced by the translation may be treated the same as the hypotheses in $E$. A subtle point here is that the translation introduces new program symbols, without adding them to the universal regular expression; however, the hypotheses added by the tranlation force the interpretations of these extra symbols to be below 1, so they could be added to the universal regular expression without affecting the validity of any formulas involved.) We then have

$$
\begin{aligned}
\mathrm{RKAT}=E \wedge r=0 \rightarrow s=t & \Rightarrow \mathrm{RKA} \models \operatorname{Tr}(E \wedge r=0 \rightarrow s=t) \\
& \Rightarrow \mathrm{RKA}=\operatorname{Tr}(E \rightarrow s+u r u=t+u r u) \\
& \Rightarrow \mathrm{RKAT} \models E \rightarrow s+u r u=t+u r u .
\end{aligned}
$$

Proof of Theorem 3.2. Immediate from Lemmas 3.3 3.5.

3.2. Idempotent Syntactic Homomorphisms. We can also eliminate hypotheses of the form $c p=c$ ( $c$ Boolean, $p$ atomic) in the presence of other hypotheses, but not as cleanly as we eliminated $r=0$ : in this case, the remaining hypotheses will be modified.

The basic idea behind the technique was introduced in [7, which showed how to simultaneously eliminate hypotheses of the form $c p=c$ and $r=0$. Ernie Cohen later observed that the portion of the proof specific to $c p=c$ was unnecessarily complicated [3]. What 
we present here is a simplified argument, that is also more general because it works in the presence of other hypotheses. Furthermore, in light of Theorem 3.2 we no longer need to worry about integrating the elimination of $r=0$ into the argument, since that can be done separately.

Definition 3.6. $H:$ RExp $_{\mathrm{P}, \mathrm{B}} \rightarrow \mathrm{RExp}_{\mathrm{P}, \mathrm{B}}$ is a syntactic homomorphism if for any interpretation $I: \operatorname{RExp}_{\mathrm{P}, \mathrm{B}} \rightarrow K$ (where $K \in \mathrm{KAT}$ ), $I \circ H: \operatorname{RExp}_{\mathrm{P} . \mathrm{B}} \rightarrow K$ is also an interpretation.

For any syntactic homomorphism $H: \operatorname{RExp}_{\mathrm{P}, \mathrm{B}} \rightarrow \operatorname{RExp}_{\mathrm{P}, \mathrm{B}}$, let $E_{H}$ be the set of hypotheses

$$
\{p=H(p) \mid p \in \mathrm{P}\} \cup\{b=H(b) \mid b \in \mathrm{B}\} .
$$

Definition 3.6 is equivalent to saying that $H$ is a homomorphism up to KAT-provable equality. A consequence is that $H$ is uniquely determined (up to KAT-provable equality) by its action on $\mathrm{P}$ and $\mathrm{B}$; the set of equations $E_{H}$ then, in a certain sense, captures the action of $H$.

(For readers familiar with guarded strings, Definition [3.6 is equivalent to saying that $G \circ H$ is an interpretation, where $G$ is the guarded-string interpretation. More abstractly, the definition is equivalent to saying that $H$ is a lift of an endomorphism on the guardedstring model - that is, there is an endomorphism $h$ on the guarded-string model such that $G \circ H=h \circ G$.)

Lemma 3.7. If $H: \operatorname{RExp}_{\mathrm{P}, \mathrm{B}} \rightarrow \operatorname{RExp}_{\mathrm{P}, \mathrm{B}}$ is a syntactic homomorphism, then for any $r \in$ RExp,$B$,

$$
\mathrm{KAT} \models E_{H} \rightarrow r=H(r) .
$$

Proof. Straightforward induction on the structure of $r$.

Definition 3.8. $H: \operatorname{RExp}_{\mathrm{P}, \mathrm{B}} \rightarrow \operatorname{RExp}_{\mathrm{P}, \mathrm{B}}$ is idempotent if for all $r \in \mathrm{RExp}_{\mathrm{P}, \mathrm{B}}$,

$$
\mathrm{KAT} \models H(r)=H(H(r)) \text {. }
$$

Theorem 3.9. Suppose $H: \operatorname{RExp}_{\mathrm{P}, \mathrm{B}} \rightarrow \mathrm{RExp}_{\mathrm{P}, \mathrm{B}}$ is an idempotent syntactic homomorphism, and that $E$ is a set of hypotheses. Let $H(E)$ denote the set of hypotheses

$$
\left\{H(r)=H\left(r^{\prime}\right) \mid r=r^{\prime} \text { is in } E\right\} .
$$

Then for any $s, t \in \mathrm{RExp}_{\mathrm{P}, \mathrm{B}}$ and $K \in \mathrm{KAT}$,

$$
K \models E \wedge E_{H} \rightarrow s=t \Longleftrightarrow K \models H(E) \rightarrow H(s)=H(t) .
$$

Proof. For the right-to-left implication, suppose $K \models H(E) \rightarrow H(s)=H(t)$ and that we have an intepretation $I: \operatorname{RExp}_{\mathrm{P}, \mathrm{B}} \rightarrow K$ with $K, I \models E \wedge E_{H}$. Then by Lemma 3.7 $K, I \models H(E) \wedge s=H(s) \wedge t=H(t)$. It follows by assumption that $K, I \models H(s)=H(t)$. We now have $K, I \models s=H(s)=H(t)=t$. Therefore, $K \models E \wedge E_{H} \rightarrow s=t$.

For the left-to-right implication, suppose $K \models E \wedge E_{H} \rightarrow s=t$, and that we have an intepretation $I: \operatorname{RExp}_{\mathrm{P}, \mathrm{B}} \rightarrow K$ with $K, I \models H(E)$. Define $I^{\prime}: \operatorname{RExp}_{\mathrm{P}, \mathrm{B}} \rightarrow K$ by $I^{\prime}=I \circ H$. $I^{\prime}$ is an interpretation by Definition 3.6. For any $p \in \mathrm{P}$, idempotence of $H$ gives us $I^{\prime}(p)=$ $I(H(p))=I(H(H(p)))=I^{\prime}(H(p))$; similarly, $I^{\prime}(b)=I^{\prime}(H(b))$ for $b \in \mathrm{B}$, so $K, I^{\prime} \models E_{H}$. For any equation $r=r^{\prime}$ in $E, K, I \models H(E)$ gives us $I^{\prime}(r)=I(H(r))=I\left(H\left(r^{\prime}\right)\right)=I^{\prime}\left(r^{\prime}\right)$, so $K, I^{\prime} \models E$. Therefore, by the assumption $K \models E \wedge E_{H} \rightarrow s=t$, we have $K, I^{\prime} \models s=t$, and hence $I(H(s))=I^{\prime}(s)=I^{\prime}(t)=I(H(t))$. Therefore $K, I \models H(s)=H(t)$, as desired. 
Corollary 3.10. Suppose $F$ is a set of hypotheses $c_{i} p_{i}=c_{i}, 1 \leq i \leq k$, where $p_{i} \in \mathrm{P}$ are distinct, and each $c_{i}$ is a Boolean term. Define $H: \operatorname{RExp}_{\mathrm{P}, \mathrm{B}} \rightarrow \operatorname{RExp}_{\mathrm{P}, \mathrm{B}}$ by $H(r)=$ $r\left[p_{i} / \overline{c_{i}} p_{i}+c_{i}\right]$, the result of substituting $\overline{c_{i}} p_{i}+c_{i}$ for $p_{i}$ in $r$ (for each $i$ ). Then for any set $E$ of hypotheses, $s, t \in \mathrm{RExp}_{\mathrm{P}, \mathrm{B}}$, and $K \in \mathrm{KAT}$, we have

$$
K \models E \wedge F \rightarrow s=t \Longleftrightarrow K \models H(E) \rightarrow H(s)=H(t) .
$$

Proof. It is easy to verify that $H$ is an idempotent syntactic homomorphism.

Next, observe that $\mathrm{KAT} \models c_{i} p_{i}=c_{i} \leftrightarrow p_{i}=\bar{c}_{i} p_{i}+c_{i}$. Every equation in $E_{H}$ is either of the form $p_{i}=\overline{c_{i}} p_{i}+c_{i}$, or is a tautology such as $b=b$, so $F$ is equivalent to $E_{H}$. The corollary now follows immediately from Theorem 3.9

The restriction that the $p_{i}$ be distinct in Corollary 3.10 is not a significant imposition, since we can combine $c_{i} p_{i}=c_{i}$ and $c_{j} p_{j}=c_{j}$, for $p_{i}=p_{j}$, into $\left(c_{i}+c_{j}\right) p_{i}=c_{i}+c_{j}$. (Supposing $c p=c$ and $d p=d$, we have $(c+d) p=c p+d p=c+d$. Supposing $(c+d) p=c+d$, we have $c \leq c+d$, so $c(c+d)=c$, giving us $c p=c(c+d) p=c(c+d)=c ; d p=d$ follows similarly. $)$

\section{Conclusion and Further Questions}

Statements about the semantics of a program can often be expressed as Horn formulas in Kleene algebra with tests, and that is our primary motivation for studying the Horn theory of Kleene algebra with tests here. Hypotheses of the form $r=0$ are of particular interest, because they can capture partial correctness assertions, which are vital to studying the semantics of imperative programs.

While the validity of Horn formulas in Kleene algebra is not in general decidable, the validity of equations is. We have shown how to eliminate hypotheses of the form $r=0$, even in the presence of other hypotheses; this allows us to extend any other technique for eliminating hypotheses to include hypotheses of the form $r=0$. We have also shown how to eliminate hypotheses of the form $c p=c$ in the presence of other hypotheses (though not as cleanly: the remaining hypotheses might be modified). This allows us to decide the validity of Horn formulas that have hypotheses of these forms.

The following are a few questions for further work. What other forms of hypotheses can be eliminated? Can they be eliminated in the presence of other hypotheses? Are there useful decision procedures for the validity of certain classes of Horn formulas that are not based on eliminating hypotheses?

\section{ACKnowledgments}

This work was supported in part by NSF grant CCR-0105586 and by ONR Grant N00014-01-1-0968. The views and conclusions contained herein are those of the author and should not be interpreted as necessarily representing the official policies or endorsements, either expressed or implied, of these organizations or the US Government.

\section{REFERENCES}

[1] Adam Barth and Dexter Kozen. Equational verification of cache blocking in LU decomposition using Kleene algebra with tests. Technical Report 2002-1865, Computer Science Department, Cornell University, June 2002.

[2] Ernie Cohen. Hypotheses in Kleene algebra. Unpublished, 1994.

[3] Ernie Cohen, 2003. Private communication.

[4] Ernie Cohen, Dexter Kozen, and Frederick Smith. The complexity of Kleene algebra with tests. Technical Report 96-1598, Computer Science Department, Cornell University, July 1996. 
[5] Chris Hardin. The Horn Theory of Relational Kleene Algebra. PhD thesis, Cornell University, 2005.

[6] Chris Hardin. Proof theory for Kleene algebra. In Proc. of the 20th Symp. on Logic in Computer Science (LICS 2005), pages 290-299, Los Alamitos, CA, June 2005. IEEE.

[7] Chris Hardin and Dexter Kozen. On the elimination of hypotheses in Kleene algebra with tests. Technical Report 2002-1879, Computer Science Department, Cornell University, October 2002.

[8] Chris Hardin and Dexter Kozen. On the complexity of the Horn theory of REL. Technical Report 2003-1896, Computer Science Department, Cornell University, May 2003.

[9] Dexter Kozen. The Design and Analysis of Algorithms. Springer-Verlag, New York, 1991.

[10] Dexter Kozen. Kleene algebra with tests. Transactions on Programming Languages and Systems, pages 427-443, 1997.

[11] Dexter Kozen. On Hoare logic and Kleene algebra with tests. Trans. Computational Logic, 1(1):60-76, July 2000.

[12] Dexter Kozen. On the complexity of reasoning in Kleene algebra. Information and Computation, 179:152-162, 2002.

[13] Dexter Kozen and Maria-Cristina Patron. Certification of compiler optimizations using Kleene algebra with tests. In J. Lloyd, V. Dahl, U. Furbach, M. Kerber, K.-K. Lau, C. Palamidessi, L. M. Pereira, Y. Sagiv, and P. J. Stuckey, editors, Proc. 1st Int. Conf. Computational Logic (CL2000), volume 1861 of Lecture Notes in Artificial Intelligence, pages 568-582, London, July 2000. Springer-Verlag.

[14] Dexter Kozen and Frederick Smith. Kleene algebra with tests: completeness and decidability. In D. van Dalen and M. Bezem, editors, Proc. 10th Int. Workshop on Computer Science Logic (CSL'96), volume 1258 of Springer-Verlag Lecture Notes in Computer Science, pages 244-259, Utrecht, The Netherlands, September 1996. 